\title{
VECTOR EXTRAPOLATION APPLIED TO TRUNCATED SINGULAR VALUE DECOMPOSITION AND TRUNCATED ITERATION
}

\author{
A. BOUHAMIDI* ${ }^{*}$ K. JBILOU ${ }^{\dagger}$, L. REICHEL ${ }^{\ddagger}, H$. SADOK$^{\S}$, AND Z. WANG
}

\begin{abstract}
This paper is concerned with the computation of accurate approximate solutions of linear systems of equations and linear least-squares problems with a very ill-conditioned matrix and error-contaminated data. The solution of this kind of problems requires regularization. Common regularization methods include the truncated singular value decomposition and truncated iteration with a Krylov subspace method. It can be difficult to determine when to truncate. Recently, it has been demonstrated that extrapolation of approximate solutions determined by truncated singular value decomposition gives a new sequence of approximate solutions that is less sensitive to the error in the data than the original approximate solutions. The present paper describes a novel approach to determine a suitable truncation index by comparing the original and extrapolated approximate solutions. Applications to truncated singular value decomposition and the LSQR iterative method are presented.
\end{abstract}

Key words. discrete ill-posed problem, vector extrapolation, truncated singular value decomposition, truncated iteration, LSQR, truncation criterion

1. Introduction. We are concerned with the computation of an approximate solution of linear least-squares problems

$$
\min _{x \in \mathbb{R}^{n}}\|A x-b\|
$$

with a matrix $A \in \mathbb{R}^{m \times n}$ of ill-determined rank, i.e., $A$ has many singular values of different orders of magnitude close to zero. In particular, $A$ is severely ill-conditioned and possibly singular. The vector $b \in \mathbb{R}^{m}$ represents available data, which is assumed to be contaminated by an error $e$. Throughout this paper $\|\cdot\|$ denotes the Euclidean vector norm or the spectral matrix norm. For ease of notation, we will assume that $m \geq n$; however, the methods discussed also are applicable when $m<n$.

Linear least-squares problems (1.1) with a matrix of ill-determined rank often are referred to as discrete ill-posed problems. They arise in science and engineering when one seeks to determine the cause of an observed effect, e.g., by remote sensing. The error in the data vector $b$ is assumed to stem from measurement inaccuracies. Let $\hat{b} \in \mathbb{R}^{m}$ denote the unknown error-free vector associated with $b$, i.e.,

$$
b=\hat{b}+e .
$$

* Laboratoire de Mathématiques Pures et Appliquées, Université du Littoral, Centre Universtaire de la Mi-Voix, Batiment H. Poincarré, 50 Rue F. Buisson, BP 699, 62228 Calais cedex, France. E-mail: bouhamidi@lmpa.univ-littoral.fr.

†Laboratoire de Mathématiques Pures et Appliquées, Université du Littoral, Centre Universtaire de la Mi-Voix, Batiment H. Poincarré, 50 Rue F. Buisson, BP 699, 62228 Calais cedex, France. E-mail: jbilou@lmpa.univ-littoral.fr.

$¥$ Department of Mathematical Sciences, Kent State University, Kent, OH 44242, USA. E-mail: reichel@math.kent.edu. Research supported in part by NSF grant DMS-1115385.

$\S$ Laboratoire de Mathématiques Pures et Appliquées, Université du Littoral, Centre Universtaire de la Mi-Voix, Batiment H. Poincarré, 50 Rue F. Buisson, BP 699, 62228 Calais cedex, France. E-mail: sadok@lmpa.univ-littoral.fr.

『Department of Mathematics, College of Science, Nanjing University of Aeronautics and Astronautics, No 29 Yudao Street, Nanjing 210016, P. R. China. E-mail: wangzhengsheng@nuaa.edu.cn. Research supported by the Jiangsu Oversea Research \& Training Program for University Prominent Young \& Middle-aged Teachers and Presidents and the Fundamental Research Funds for the Central Universities grant NZ2012307. 
We are interested in determining an accurate approximation of the solution $\hat{x}$ of minimal Euclidean norm of the unavailable least-squares problem

$$
\min _{x \in \mathbb{R}^{n}}\|A x-\hat{b}\|
$$

with error-free data vector. Thus, $\hat{x}=A^{\dagger} \hat{b}$, where $A^{\dagger}$ denotes the Moore-Penrose pseudoinverse of $A$. Note that due to the error $e$ in $b$ and the ill-conditioning of the matrix $A$, the vector

$$
A^{\dagger} b=A^{\dagger}(\hat{b}+e)=\hat{x}+A^{\dagger} e
$$

generally does not furnish a meaningful approximation of $\hat{x}$ due to a large propagated error $A^{\dagger} e$.

A popular method for computing an approximation of $\hat{x}$ when $A$ is of small to moderate size is to use the truncated singular value decomposition (TSVD). This method replaces $A$ and $A^{\dagger}$ by low-rank approximations and thereby reduces the size of the propagated error; see, e.g., [1,2] for discussions. A brief review of this method is provided in Section 2. A difficulty when applying the TSVD method is to determine a suitable rank of the approximation of $A$. The rank, which generally is equal to the truncation index, can be considered a regularization parameter. This parameter determines how much the problem actually solved differs from the given problem (1.1) and how sensitive the computed solution is to the error $e$ in $b$ and to round-off errors introduced during the computation.

Vector extrapolation can be applied to the approximate solutions determined by TSVD. Computed examples reported in $[3,4]$ show the extrapolated approximate solutions to be less sensitive to the error $e$ in the data $b$ than the approximate solutions determined by standard TSVD when the truncation index is larger than the value that gives the best approximation of $\hat{x}$. This paper proposes to determine the truncation index for TSVD based on the difference between the standard and extrapolated approximate solutions. This way of determining a suitable truncation index does not require an estimate of the norm of the error $e$.

Rules for determining a suitable truncation index without explicitly using an estimate of $\|e\|$ are often referred to as "heuristic"; see, e.g., $[1,5]$. Commonly used heuristic rules include the L-curve criterion, the quasi-optimality criterion, and generalized cross validation. All heuristic rules are known to sometimes fail to determine a suitable truncation index; see, e.g., Kindermann [5] for a recent discussion. Nevertheless, the development of heuristic rules is important, because in many applications where linear discrete ill-posed problems arise no useful estimate of $\|e\|$ is known; see, e.g., $[5,6,7]$ and references therein for recent discussions on heuristic methods.

The application of extrapolation methods to the solution of linear discrete illposed problems was first proposed by Brezinski et al. [8]; see also [9, 10, 11] for more recent discussions. A nice survey of extrapolation methods is provided by Brezinski and Redivo Zaglia [12]. Discussion on vector extrapolation and applications can be found in $[13,14]$.

LSQR [15] is a popular iterative Krylov subspace method for the approximate solution of linear discrete ill-posed problems (1.1) when the matrix $A$ is too large for fast evaluation of its SVD; see Engl et al. [1, Chapter 7] for a discussion of this application. LSQR determines a sequence of approximate solutions. Similarly as for the TSVD, the determination of a suitable truncation index is important for the quality of the computed approximate solutions. We propose to compare standard and extrapolated LSQR iterates to determine when to terminate the iterations. 
This paper is organized as follows. Section 2 reviews TSVD and reduced rank extrapolation (RRE), and discusses the application of the latter to the sequence of approximate solutions determined by the former. Section 3 outlines the LSQR iterative method and describes the application of RRE to the iterates computed by LSQR. A few computed examples are presented in Section 4 and concluding remarks can be found in Section 5.

2. TSVD and RRE. This section reviews the TSVD method, RRE, and the application of RRE to the sequence of approximate solutions determined by TSVD.

2.1. The TSVD method. Introduce the singular value decomposition

$$
A=\sum_{j=1}^{n} \sigma_{j} u_{j} v_{j}^{T}
$$

with the singular values $\sigma_{j}$ ordered so that

$$
\sigma_{1} \geq \sigma_{2} \geq \ldots \geq \sigma_{r}>\sigma_{r+1}=\ldots=\sigma_{n}=0,
$$

where $r$ is the rank of $A$. Then

$$
A v_{j}=\sigma_{j} u_{j}, \quad A^{T} u_{j}=\sigma_{j} v_{j}, \quad 1 \leq j \leq n,
$$

and the matrices $U=\left[u_{1}, u_{2}, \ldots, u_{n}\right] \in \mathbb{R}^{m \times n}$ and $V=\left[v_{1}, v_{2}, \ldots, v_{n}\right] \in \mathbb{R}^{n \times n}$ have orthonormal columns, see, e.g., $[2,16]$ for details on the singular value decomposition. Typically, many of the smallest nonvanishing singular values of the matrix of discrete ill-posed problems (1.1) are tiny.

Let $1 \leq k \leq r$. The best rank- $k$ approximation of $A$ in the spectral norm is given by

$$
A_{k}=\sum_{j=1}^{k} \sigma_{j} u_{j} v_{j}^{T} .
$$

It is well known that, for $1 \leq k<n$,

$$
\left\|A_{k}-A\right\|=\sigma_{k+1} .
$$

Thus, the larger $k$, the better $A_{k}$ approximates $A$. The Moore-Penrose pseudoinverse of $A_{k}$ for $1 \leq k \leq r$ can be written as

$$
A_{k}^{\dagger}=\sum_{j=1}^{k} \sigma_{j}^{-1} v_{j} u_{j}^{T} .
$$

The solution $x_{k}$ of minimal Euclidean norm of the least-squares problem

$$
\min _{x \in \mathbb{R}^{n}}\left\|A_{k} x-b\right\|
$$

can be conveniently expressed with the aid of $A_{k}^{\dagger}$. We have, for $1 \leq k \leq r$,

$$
x_{k}:=A_{k}^{\dagger} b=\sum_{j=1}^{k} \frac{u_{j}^{T} b}{\sigma_{j}} v_{j} .
$$


The propagated error in $x_{k}$ is given by $\sum_{j=1}^{k} \frac{u_{j}^{T} e}{\sigma_{j}} v_{j}$. The norm of the propagated error increases with $k$. It is therefore prudent not to choose the truncation index $k$ too large. However, for a small value of $k$ the truncation error (2.1) may be large and the problem solved (2.2) may differ significantly from the given discrete ill-posed problem (1.1). Therefore, it is important to choose the truncation index $k$ to be neither too large nor too small.

We would like to determine an index $k$, such that $x_{k}$ defined by (2.3) is an accurate approximation of $\hat{x}$. This will be done with the aid of vector extrapolation, which is reviewed in the following subsection. For future reference, we define $k_{\mathrm{opt}} \geq 1$ to be the smallest integer such that

$$
\left\|x_{k_{\mathrm{opt}}}-\hat{x}\right\|=\min _{k \geq 1}\left\|x_{k}-\hat{x}\right\| .
$$

2.2. RRE. The convergence of a sequence of slowly converging vectors often can be accelerated by extrapolation methods. Reduced rank extrapolation (RRE) by Eddy [17] and Mesina [18] is a popular vector extrapolation method. Let

$$
s_{0}, s_{1}, s_{2}, \ldots
$$

be a sequence of vectors in $\mathbb{R}^{n}$, and introduce the first and second forward differences

$$
\Delta s_{j}:=s_{j+1}-s_{j} \quad \text { and } \quad \Delta^{2} s_{j}:=\Delta s_{j+1}-\Delta s_{j} .
$$

The RRE method applied to the sequence (2.5) produces approximations $t_{k}$ of the limit or antilimit of the $s_{j}$ as $j \rightarrow \infty$ of the form

$$
t_{k}=\sum_{j=0}^{k} \gamma_{j}^{(k)} s_{j}
$$

where

$$
\sum_{j=0}^{k} \gamma_{j}^{(k)}=1 \quad \text { and } \quad \sum_{j=0}^{k} \eta_{i j} \gamma_{j}^{(k)}=0, \quad 0 \leq i<k,
$$

with $\eta_{i j}:=\left(y_{i+1}, \Delta s_{j}\right)$ and

$$
y_{i+1}:=\Delta^{2} s_{i}
$$

Define the matrices

$$
Y_{k}:=\left[y_{1}, y_{2}, \ldots, y_{k}\right], \quad \Delta^{i} S_{k}:=\left[\Delta^{i} s_{0}, \Delta^{i} s_{1}, \ldots, \Delta^{i} s_{k-1}\right], \quad i=1,2 .
$$

Using Schur's formula, the vector $t_{k}$ can be expressed as

$$
t_{k}=s_{0}-\Delta S_{k}\left(Y_{k}^{T} \Delta^{2} S_{k}\right)^{-1} Y_{k}^{T} \Delta s_{0},
$$

where we remark that $t_{k}$ exists and is unique if and only if $\operatorname{det}\left(Y_{k}^{T} \Delta^{2} S_{k}\right) \neq 0$. Further manipulations, described in, e.g., [4], show that

$$
t_{k}=s_{0}+\sum_{j=0}^{k-1} \alpha_{j}^{(k)} \Delta s_{j}
$$

for certain coefficients $\alpha_{j}^{(k)}$. Algorithms by Ford and Sidi [19] can be applied to the computation of the $t_{k}, k=0,1,2, \ldots$. Details of these computations and additional references can be found in [4]. 
2.3. Application of RRE to TSVD. Let the vectors $y_{i}$ be defined by (2.6) and let the $s_{k}$ be the TSVD solutions (2.3), i.e.,

$$
s_{0}:=0, \quad s_{k}:=x_{k}=A_{k}^{\dagger} b=\sum_{j=1}^{k} \frac{u_{j}^{T} b}{\sigma_{j}} v_{j}=\sum_{j=1}^{k} \delta_{j} v_{j},
$$

where $\delta_{j}:=u_{j}^{T} b / \sigma_{j}$. This yields

$$
\Delta s_{k-1}=s_{k}-s_{k-1}=\delta_{k} v_{k} .
$$

We may assume that $\delta_{k} \neq 0$, because otherwise we delete the corresponding member from the sequence (2.9) and compute the next one by keeping the same index notation. The matrix $\Delta S_{k}=\left[\Delta s_{0}, \Delta s_{1}, \ldots, \Delta s_{k-1}\right]$ can be factored according to

$$
\Delta S_{k}=\left[\delta_{1} v_{1}, \delta_{2} v_{2} \ldots, \delta_{k} v_{k}\right]=V_{k} \operatorname{diag}\left[\delta_{1}, \delta_{2}, \ldots, \delta_{k}\right],
$$

where $V_{k}=\left[v_{1}, v_{2}, \ldots, v_{k}\right]$. Since $\Delta^{2} s_{k-1}=\delta_{k+1} v_{k+1}-\delta_{k} v_{k}$, we obtain that

$$
\Delta^{2} S_{k}=V_{k+1}\left[\begin{array}{cccc}
-\delta_{1} & & & \\
\delta_{2} & -\delta_{2} & & \\
& \ddots & \ddots & \\
& & \delta_{k} & -\delta_{k} \\
& & & \delta_{k+1}
\end{array}\right]
$$

and (2.11) yields

$$
\Delta^{2} S_{k}^{T} \Delta S_{k}=\left[\begin{array}{cccc}
-\delta_{1} & & & \\
\delta_{2} & -\delta_{2} & & \\
& \ddots & \ddots & \\
& & \delta_{k} & -\delta_{k} \\
& & & \delta_{k+1}
\end{array}\right]^{T} V_{k+1}^{T}\left[\delta_{1} v_{1}, \delta_{2} v_{2} \ldots, \delta_{k} v_{k}\right] .
$$

In view of that

$$
V_{k+1}^{T}\left[\delta_{1} v_{1}, \delta_{2} v_{2}, \ldots, \delta_{k} v_{k}\right]=\operatorname{diag}\left[\delta_{1}, \delta_{2}, \ldots, \delta_{k}\right] \in \mathbb{R}^{(k+1) \times k},
$$

we have

$$
\Delta^{2} S_{k}^{T} \Delta S_{k}=\left[\begin{array}{ccccc}
-\delta_{1}^{2} & \delta_{2}^{2} & & & \\
& -\delta_{2}^{2} & \delta_{3}^{2} & & \\
& & \ddots & \ddots & \\
& & & -\delta_{k-1}^{2} & \delta_{k}^{2} \\
& & & & -\delta_{k}^{2}
\end{array}\right]
$$

and application of (2.10) and (2.11) yields

$$
\Delta^{2} S_{k}^{T} \Delta s_{k}=\left[0, \ldots, 0, \delta_{k+1}^{2}\right]^{T} .
$$


The above relations can be applied to derive an expression for $t_{k}$ in (2.7). In particular, we obtain that the scalars $\alpha_{0}^{(k)}, \alpha_{1}^{(k)}, \ldots, \alpha_{k-1}^{(k)}$ in (2.8) are given by

$$
\alpha_{i}^{(k)}=\frac{\sum_{j=i+1}^{k} \frac{1}{\delta_{j+1}^{2}}}{\sum_{l=0}^{k} \frac{1}{\delta_{l+1}^{2}}}, \quad 0 \leq i<k
$$

It follows that the vector $t_{k}$ can be written as

$$
t_{k}=\sum_{j=1}^{k} \alpha_{j-1}^{(k)} \frac{u_{j}^{T} b}{\sigma_{j}} v_{j}
$$

which shows that applying RRE to TSVD solutions corresponds to using the filter factors $\alpha_{j-1}^{(k)}$.

The computations for the RRE-TSVD method are summarized by the following algorithm:

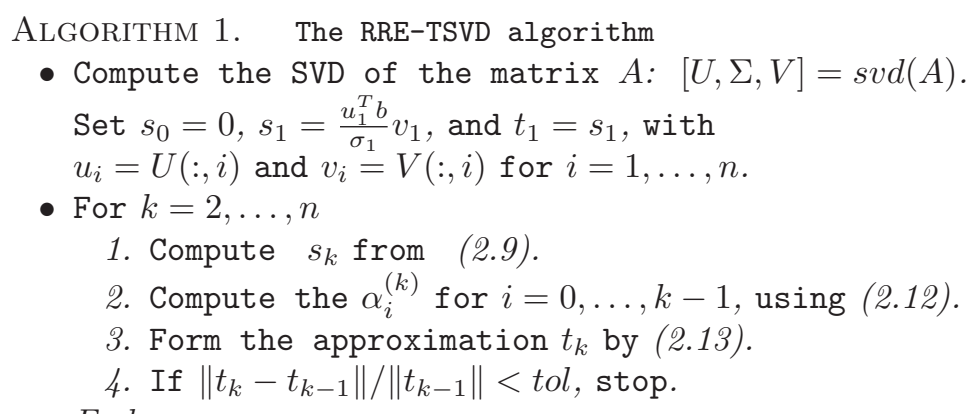

In the computed examples of Section 4, we let the parameter tol be eps in MATLAB, i.e., about $2.22 \times 10^{-16}$.

3. Extrapolated LSQR. LSQR is a popular iterative method for the solution of large discrete ill-posed problems of the form (1.1). Let the initial iterate be $x_{0}=0$. Then the $k$ th iterate, $x_{k}$, determined by LSQR applied to the solution of (1.1) is computed with the aid of the partial Golub-Kahan bidiagonalization

$$
A \widetilde{V}_{k}=\widetilde{U}_{k+1} \widetilde{B}_{k+1, k}, \quad A^{T} \widetilde{U}_{k}=\widetilde{V}_{k} \widetilde{B}_{k}^{T}
$$

where the matrices $\widetilde{U}_{k+1} \in \mathbb{R}^{m \times(k+1)}$ and $\widetilde{V}_{k} \in \mathbb{R}^{n \times k}$ have orthonormal columns, $\widetilde{U}_{k} \in \mathbb{R}^{m \times k}$ consists of the first $k$ columns of $\widetilde{U}_{k+1}$, the first column of $\widetilde{U}_{k+1}$ is $b /\|b\|$, and $\widetilde{B}_{k+1, k} \in \mathbb{R}^{(k+1) \times k}$ is lower bidiagonal with leading submatrix $\widetilde{B}_{k} \in \mathbb{R}^{k \times k}$. The iterate $x_{k}$ is determined by minimizing the residual error over the Krylov subspace

$$
\operatorname{range}\left(\widetilde{V}_{k}\right)=\mathcal{K}_{k}\left(A^{T} A, A^{T} b\right):=\operatorname{span}\left\{A^{T} b,\left(A^{T} A\right) A^{T} b, \ldots,\left(A^{T} A\right)^{k-1} A^{T} b\right\}
$$

Thus, $x_{k} \in \mathcal{K}_{k}\left(A^{T} A, A^{T} b\right)$ satisfies

$$
\left\|A x_{k}-b\right\|=\min _{y \in \mathbb{R}^{k}}\left\|A \widetilde{V}_{k} y-b\right\|=\min _{y \in \mathbb{R}^{k}}\left\|\widetilde{U}_{k+1} \widetilde{B}_{k+1, k} y-b\right\|=\min _{y \in \mathbb{R}^{k}}\left\|\widetilde{B}_{k+1, k} y-\right\| b\left\|e_{1}\right\|,
$$


where $e_{1}=[1,0, \ldots, 0]^{T} \in \mathbb{R}^{k+1}$ denotes the first axis vector. Let $y_{k}$ be the solution of the (small) minimization problem on the right-hand side. Then $x_{k}=V_{k} y_{k}$; see [15] for details. Here we have assumed that $k$ is small enough so that the matrix $\widetilde{B}_{k+1, k}$ is of full rank.

The LSQR method updates successive iterates according to

$$
x_{k}=x_{k-1}+d_{k}
$$

and the differences $d_{k}$ between successive iterates are available during the execution of LSQR. We define the sequence

$$
s_{0}:=x_{0}=0, \quad s_{k}:=x_{k}, \quad k=1,2,3, \ldots .
$$

Then

$$
\Delta s_{k}=s_{k+1}-s_{k}=d_{k}, \quad \Delta^{2} s_{k}=\Delta s_{k+1}-\Delta s_{k}=d_{k+1}-d_{k} .
$$

We can now apply RRE to determine an extrapolated sequence $t_{0}, t_{1}, t_{2}, \ldots$ similarly as in Section 2.

4. Numerical examples. All computations are carried out using MATLAB version 7.4 with machine epsilon $\epsilon \approx 2.22 \times 10^{-16}$. The examples stem from the MATLAB package Regularization Tools by Hansen [20]. The matrices $A$ and the desired solutions $\hat{x}$ are determined by codes from this package; all matrices are discretizations of compact integral operators with a smooth kernel. The (assumed unknown) errorfree data vector is given by $\hat{b}:=A \hat{x}$ and the associated error-contaminated vector $b$ is determined by (1.2), where the "error vector" $e$ has normally distributed entries with zero mean. The vector $e$ is normalized to correspond to a specified noise-level

$$
\nu=\frac{\|e\|}{\|\hat{b}\|} .
$$

In this section, we denote the sequence of approximate solutions determined by TSVD or LSQR by $x_{0}, x_{1}, x_{2}, \ldots$ and the associated vector sequence computed by RRE by $t_{0}, t_{1}, t_{2}, \ldots$.

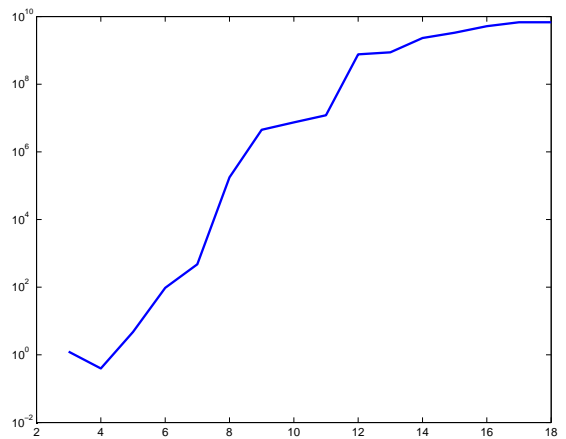

(a)

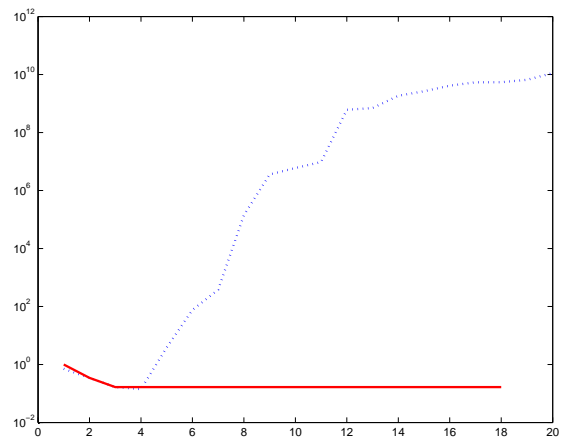

(b)

FIG. 4.1. Example 4.1: Noise-level $\nu=1 \times 10^{-2}$. (a) Differences $\left\|t_{k}-x_{k}\right\|$ for $k=2,3,4, \ldots$. (b) Relative errors $\left\|x_{k}-\hat{x}\right\| /\|\hat{x}\|$ for TSVD (blue dashed curve) and relative errors $\left\|t_{k}-\hat{x}\right\| /\|\hat{x}\|$ for RRE-TSVD (red solid curve). 


\begin{tabular}{ccc}
$k$ & $\left\|x_{k}-\hat{x}\right\| /\|\hat{x}\|$ & $\left\|t_{k}-\hat{x}\right\| /\|\hat{x}\|$ \\
\hline 3 & $1.67 \times 10^{-1}$ & $1.24 \times 10^{0}$ \\
4 & $1.42 \times 10^{-1}$ & $3.96 \times 10^{-1}$ \\
5 & $3.89 \times 10^{0}$ & $4.75 \times 10^{0}$ \\
& \multicolumn{2}{c}{ TABLE 4.1}
\end{tabular}

Example 4.1: Noise-level $\nu=1 \times 10^{-2}$. Errors in TSVD solutions $x_{k}$ and in extrapolated TSVD solutions $t_{k}$.

Example 4.1. We illustrate the behavior of the sequence $x_{1}, x_{2}, x_{3}, \ldots$ of approximate solutions of (1.1) determined by TSVD and of the sequence $t_{1}, t_{2}, t_{3}, \ldots$ obtained by reduced rank extrapolation of the former sequence. The discrete ill-posed problem solved is obtained by discretizing the integral equation

$$
\int_{0}^{\pi / 2} \kappa(s, t) x(t) \mathrm{d} t=g(s), \quad 0 \leq s \leq \pi,
$$

where

$$
\kappa(s, t)=\exp (s \cos (t)) \text { and } g(s)=2 \sin (s) / s .
$$

The solution is given by $x(t)=\sin (t)$. This integral equation is discussed by Baart [21]. We used the MATLAB code baart from [20] to discretize (4.1) by a Galerkin method with 1000 orthonormal box functions as test and trial functions. This yields the nonsymmetric matrix $A \in \mathbb{R}^{1000 \times 1000}$ and the vector $\hat{x} \in \mathbb{R}^{1000}$, which is a scaled discretization of $x(t)$. The condition number of $A$, defined by $\kappa(A):=\|A\|\left\|A^{-1}\right\|$, is computed with the MATLAB function cond and found to be about $2 \times 10^{19}$. Thus, the matrix is numerically singular. It has many nonvanishing singular values close to zero. The error-contaminated vector $b \in \mathbb{R}^{1000}$ corresponds to the noise-level $\nu=1 \times 10^{-2}$.

Figure 4.1(a) displays the norm of the differences $\left\|t_{k}-x_{k}\right\|, k=2,3, \ldots$. Note the logarithmic scale. Our selection criterion for the truncation index is to choose the index for which $\left\|t_{k}-x_{k}\right\|$ is the smallest. In case of nonunicity, we choose the smallest index $k$ with this property. We refer to this approach to choosing the truncation index as the extrapolation criterion and denote the index so determined by $k_{\text {extrapol }}$. Thus, $k_{\text {extrapol }}=4$ for the present example.

Table 4.1 shows that $x_{4}$, indeed, provides the most accurate approximation of $\hat{x}$. Figure $4.1(\mathrm{~b})$ depicts the relative errors $\left\|x_{k}-\hat{x}\right\| /\|\hat{x}\|$ in the TSVD solutions as a function of $k$ (blue dashed curve) and in the extrapolated TSVD solutions $\left\|t_{k}-\hat{x}\right\| /\|\hat{x}\|$ (red solid curve). The latter relative errors are seen not to grow when $k$ increases. This implies that it is less crucial to determine the best truncation index when the approximate solutions $t_{k}$ of (1.1) are used. However, Figure 4.1(b) as well as Table 4.1 show the error in $x_{4}$ to be smaller than the error in $t_{4}$. It is clear from Figure 4.1 (b) that $k_{\text {opt }}$ defined by (2.4) has the value 4 .

Example 4.2. We generate the nonsymmetric matrix $A \in \mathbb{R}^{1000 \times 1000}$ and the desired solution $\hat{x} \in \mathbb{R}^{1000}$ with the code wing from [20]; see [22] for a description of this problem. The matrix $A$ is numerically singular and has many "tiny" singular values. The vector $b$ is generated similarly as in Example 4.1 with noise-level $\nu=1 \times 10^{-2}$.

Figure 4.2(a) shows the norm of the differences between the approximate solutions $x_{k}$ determined by TSVD and the corresponding extrapolated approximate solutions $t_{k}$. The smallest difference is achieved for $k=4$. Thus, our extrapolation criterion determines the truncation index $k_{\text {extrapol }}=4$ and we choose the approximate solution 


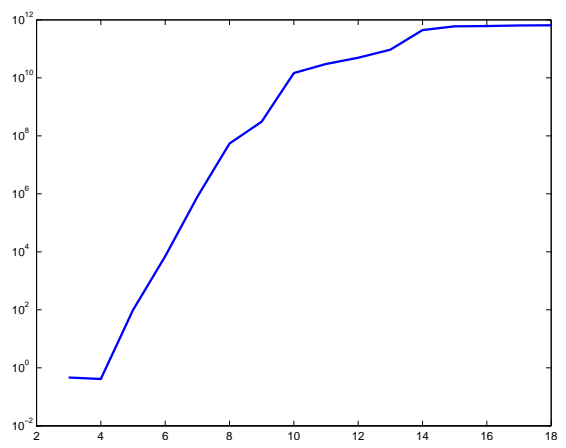

(a)

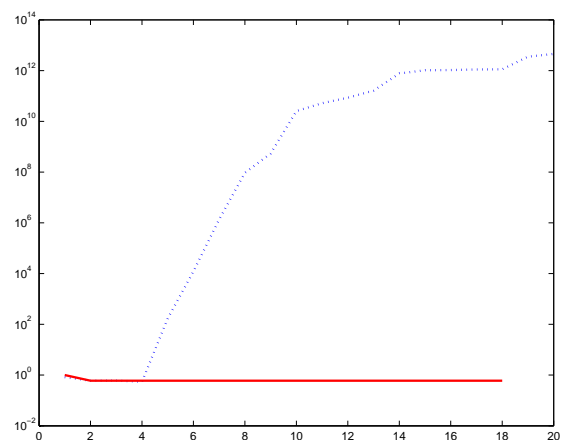

(b)

Fig. 4.2. Example 4.2: Noise-level $\nu=1 \times 10^{-2}$. (a) Differences $\left\|t_{k}-x_{k}\right\|$ for $k=2,3,4, \ldots$. (b) Relative errors $\left\|x_{k}-\hat{x}\right\| /\|\hat{x}\|$ for TSVD (blue dashed curve) and relative errors $\left\|t_{k}-\hat{x}\right\| /\|\hat{x}\|$ for RRE-TSVD (red solid curve).

\begin{tabular}{ccc}
$k$ & $\left\|x_{k}-\hat{x}\right\| /\|\hat{x}\|$ & $\left\|t_{k}-\hat{x}\right\| /\|\hat{x}\|$ \\
\hline 3 & $6.07 \times 10^{-1}$ & $6.02 \times 10^{-1}$ \\
4 & $6.02 \times 10^{-1}$ & $6.02 \times 10^{-1}$ \\
5 & $1.70 \times 10^{+2}$ & $6.02 \times 10^{-1}$
\end{tabular}

Example 4.2: Noise-level $\nu=1 \times 10^{-2}$. Errors in TSVD solutions $x_{k}$ and in extrapolated TSVD solutions $t_{k}$.

$x_{4}$ of (1.1). Figure 4.2(b) displays the relative errors $\left\|x_{k}-\hat{x}\right\| /\|\hat{x}\|$ and $\left\|t_{k}-\hat{x}\right\| /\|\hat{x}\|$. They are small for $k=4$. Moreover, note that the errors $\left\|t_{k}-\hat{x}\right\| /\|\hat{x}\|$ do not grow as $k$ increases, while the errors $\left\|x_{k}-\hat{x}\right\| /\|\hat{x}\|$ grow quickly with $k$ for $k>4$. Table 4.2 shows $x_{4}$ to be the most accurate approximation of $\hat{x}$, i.e., $k_{\mathrm{opt}}=4$. In this example $x_{4}$ is very close to $t_{4}$ and the latter vector also could have been chosen as an approximation of $\hat{x}$.

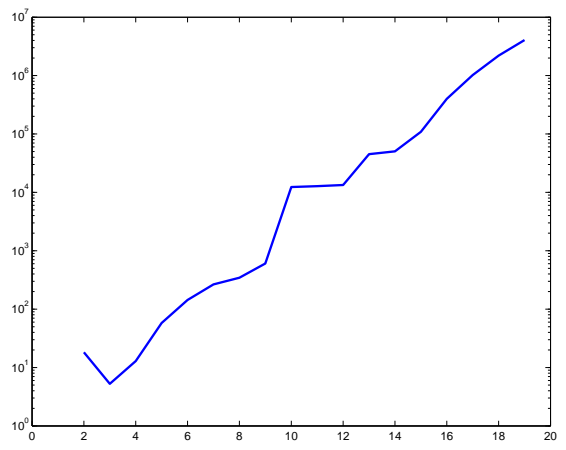

(a)

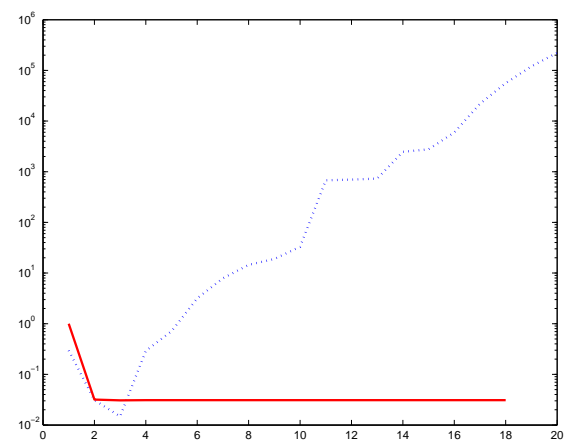

(b)

FIG. 4.3. Example 4.3: Noise-level $\nu=1 \times 10^{-2}$. (a) Differences $\left\|t_{k}-x_{k}\right\|$ for $k=2,3,4, \ldots$. (b) Relative errors $\left\|x_{k}-\hat{x}\right\| /\|\hat{x}\|$ for TSVD (blue dashed curve) and relative errors $\left\|t_{k}-\hat{x}\right\| /\|\hat{x}\|$ for RRE-TSVD (red solid curve).

Example 4.3. The MATLAB code foxgood from [20] is used to determine a linear discrete ill-posed problems (1.1) with a symmetric matrix $A \in \mathbb{R}^{1000 \times 1000}$ and 


\begin{tabular}{ccc}
$k$ & $\left\|x_{k}-\hat{x}\right\| /\|\hat{x}\|$ & $\left\|t_{k}-\hat{x}\right\| /\|\hat{x}\|$ \\
\hline 2 & $3.11 \times 10^{-2}$ & $3.18 \times 10^{-2}$ \\
3 & $1.48 \times 10^{-2}$ & $3.07 \times 10^{-2}$ \\
4 & $2.91 \times 10^{-2}$ & $3.10 \times 10^{-2}$ \\
& \multicolumn{2}{c}{ TABLE 4.3}
\end{tabular}

Example 4.3: Noise-level $\nu=1 \times 10^{-2}$. Errors in TSVD solutions $x_{k}$ and in extrapolated TSVD solutions $t_{k}$.

the solution $\hat{x} \in \mathbb{R}^{1000}$. The vectors $\hat{b} \in \mathbb{R}^{1000}$ and $b \in \mathbb{R}^{1000}$ are generated similarly as in Example 4.1. The noise-level is $\nu=1 \times 10^{-2}$. The problem is described in [23, p. 665].

Figure 4.3(a) shows the norm of the differences between the TSVD approximate solutions $x_{k}$ and the corresponding extrapolated approximate solutions $t_{k}$. The difference is smallest for $k=3$, i.e., our extrapolation criterion yields the truncation index $k_{\text {extrapol }}=3$. Figure $4.3(\mathrm{~b})$ shows the relative errors $\left\|x_{k}-\hat{x}\right\| /\|\hat{x}\|$ and $\left\|t_{k}-\hat{x}\right\| /\|\hat{x}\|$. The smallest error is achieved by $x_{3}$.

Similarly as in the above examples, the errors $\left\|t_{k}-\hat{x}\right\| /\|\hat{x}\|$ do not grow as $k$ increases, while the errors $\left\|x_{k}-\hat{x}\right\| /\|\hat{x}\|$ grow rapidly with $k$ for $k>3$. This is an attractive feature of the extrapolated approximate solutions $t_{k}$. However, Table 4.3 shows $x_{3}$ to be a more accurate approximation of $\hat{x}$ than $t_{3}$. We have for many discrete ill-posed problems observed that $\left\|x_{k_{\text {extrapol }}}-\hat{x}\right\|<\left\|t_{k_{\text {extrapol }}}-\hat{x}\right\|$. Therefore, we typically will select $x_{k_{\text {extrapol }}}$ as our approximation of $\hat{x}$ and not $t_{k_{\text {extrapol }}}$. Figure 4.3(b) and Table 4.3 show that $k_{\mathrm{opt}}=3$.

In the remaining examples we illustrate the performance of the extrapolationbased stopping criterion when the approximate solutions $x_{1}, x_{2}, x_{3}, \ldots$ are iterates determined by LSQR. The corresponding extrapolated vectors are denoted by

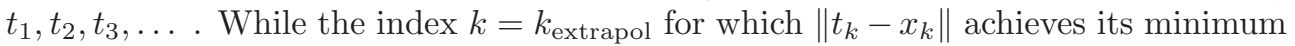
is straightforward to determine when TSVD is applied, it is more difficult when we use LSQR iterates and the associated extrapolated vectors. The reason for this is that we would like to avoid to carry out many more than $k_{\text {extrapol }}$ steps with LSQR. We have found it to be sufficient to carry out 5 iterations after the last computed local minimum of $k \rightarrow\left\|t_{k}-x_{k}\right\|$. If no new local minimum is detected during these 5 steps, then we terminate the iterations; otherwise we carry out 5 iterations after the last detected local minimum. In all our experiments this has resulted in the correct identification of the global minimum and thereby of $k_{\text {extrapol}}$.

\begin{tabular}{ccc}
$k$ & $\left\|x_{k}-\hat{x}\right\| /\|\hat{x}\|$ & $\left\|t_{k}-\hat{x}\right\| /\|\hat{x}\|$ \\
\hline 9 & $5.18 \times 10^{-2}$ & $5.36 \times 10^{-2}$ \\
10 & $5.16 \times 10^{-2}$ & $5.34 \times 10^{-2}$ \\
11 & $5.19 \times 10^{-2}$ & $5.36 \times 10^{-2}$ \\
& \multicolumn{2}{c}{ TABLE 4.4}
\end{tabular}

Example 4.4: Noise-level $\nu=1 \times 10^{-2}$. Errors in LSQR solutions $x_{k}$ and in extrapolated $L S Q R$ solutions $t_{k}$.

Example 4.4. The present example determines the linear discrete ill-posed problems and the desired solution $\hat{x}$ with the code shaw from [20]. The symmetric matrix $A \in \mathbb{R}^{1000 \times 1000}$ obtained is of ill-determined rank. A description of the integral operator of which $A$ is a discretization is provided by Shaw [24]. The vector $b \in \mathbb{R}^{1000}$ is generated analogously as in the previous example. 


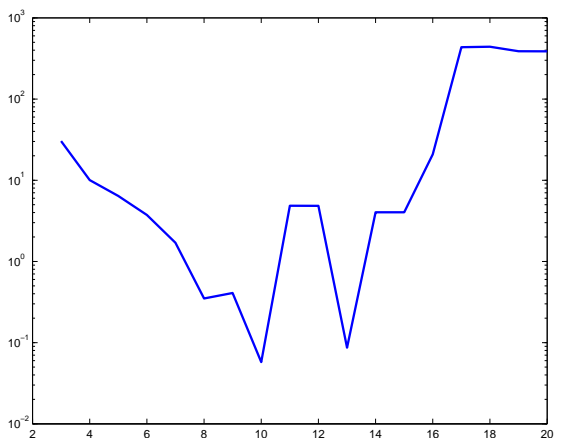

(a)

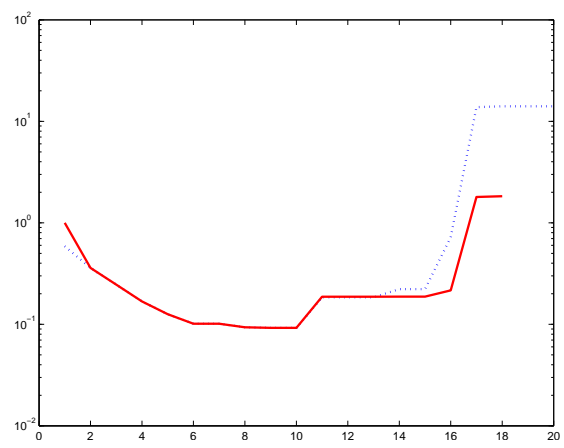

(b)

FIG. 4.4. Example 4.4: Noise-level $\nu=1 \times 10^{-2}$. (a) Differences $\left\|t_{k}-x_{k}\right\|$ for $k=2,3,4, \ldots$. (b) Relative errors $\left\|x_{k}-\hat{x}\right\| /\|\hat{x}\|$ for LSQR (blue dashed curve) and relative errors $\left\|t_{k}-\hat{x}\right\| /\|\hat{x}\|$ for $R R E-L S Q R$ (red solid curve).

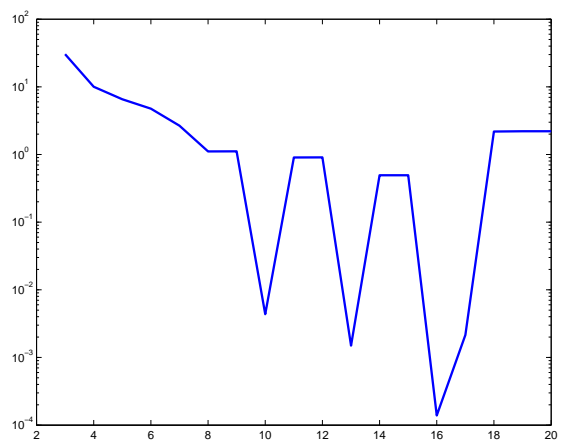

(a)

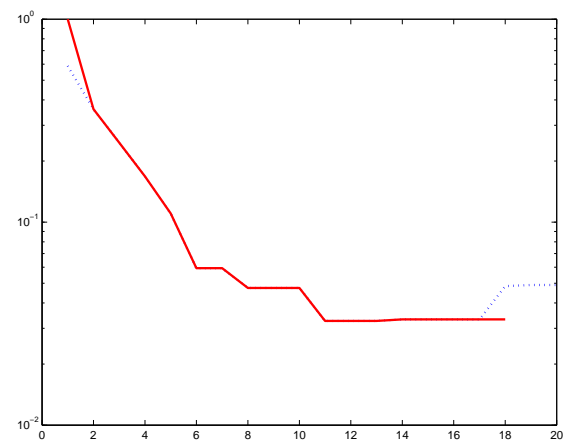

(b)

Fig. 4.5. Example 4.4: Noise-level $\nu=1 \times 10^{-4}$. (a) Differences $\left\|t_{k}-x_{k}\right\|$ for $k=2,3,4, \ldots$. (b) Relative errors $\left\|x_{k}-\hat{x}\right\| /\|\hat{x}\|$ for LSQR (blue dashed curve) and relative errors $\left\|t_{k}-\hat{x}\right\| /\|\hat{x}\|$ for RRE-LSQR (red solid curve).

We first consider the case when the noise-level is $\nu=1 \times 10^{-2}$. Figure 4.4(a) displays the norm of the differences $\left\|x_{k}-t_{k}\right\|$. The graph shows that $k_{\text {extrapol }}=10$, and Figure 4.4(b), which displays the relative errors $\left\|x_{k}-\hat{x}\right\| /\|\hat{x}\|$ and $\left\|t_{k}-\hat{x}\right\| /\|\hat{x}\|$, confirms that $x_{10}$ is an accurate approximation of $\hat{x}$. Table 4.4 shows the difference $x_{k}-\hat{x}$ to be nearly minimal for $k=10$. In this example both the errors vectors $x_{k}-\hat{x}$ and $t_{k}-\hat{x}$ increase as $k \geq 10$ increases.

We now reduce the noise-level to $\nu=1 \times 10^{-4}$. Figure 4.5 and Table 4.5 are analogous to Figure 4.4 and Table 4.4, respectively, for this smaller noise-level. Figure 4.5 (a) shows the extrapolation criterion to yield the truncation index $k_{\text {extrapol }}=16$ and Figure $4.5(\mathrm{~b})$ shows both the relative errors $\left\|x_{16}-\hat{x}\right\| /\|\hat{x}\|$ and $\left\|t_{16}-\hat{x}\right\| /\|\hat{x}\|$ to be small. Table 4.5 confirms that the truncation index $k=16$ is a good choice both for the iterates $x_{k}$ and the extrapolated iterates $t_{k}$.

Example 4.5. This example is generated in the same way as Example 4.1, i.e., we apply the code baart from [20] to determine the nonsymmetric matrix $A \in \mathbb{R}^{1000 \times 1000}$ and solution $\hat{x} \in \mathbb{R}^{1000}$. The vector $b \in \mathbb{R}^{1000}$ is evaluated similarly as in Example 4.1. The noise-level is $\nu=1 \times 10^{-2}$.

Figure 4.6(a) displays the difference between the LSQR iterates $x_{k}$ and the cor- 


\begin{tabular}{ccc}
$k$ & $\left\|x_{k}-\hat{x}\right\| /\|\hat{x}\|$ & $\left\|t_{k}-\hat{x}\right\| /\|\hat{x}\|$ \\
\hline 15 & $3.32 \times 10^{-2}$ & $3.32 \times 10^{-2}$ \\
16 & $3.31 \times 10^{-2}$ & $3.32 \times 10^{-2}$ \\
17 & $3.32 \times 10^{-2}$ & $3.32 \times 10^{-2}$ \\
18 & $4.84 \times 10^{-2}$ & $3.32 \times 10^{-2}$ \\
19 & $4.90 \times 10^{-2}$ & $3.32 \times 10^{-2}$ \\
\multicolumn{3}{c}{ TABLE 4.5}
\end{tabular}

Example 4.4: Noise-level $\nu=1 \times 10^{-4}$. Errors in LSQR solutions $x_{k}$ and in extrapolated LSQR solutions $t_{k}$.

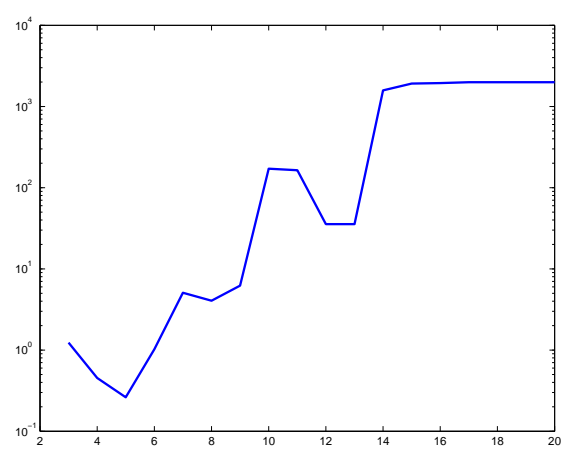

(a)

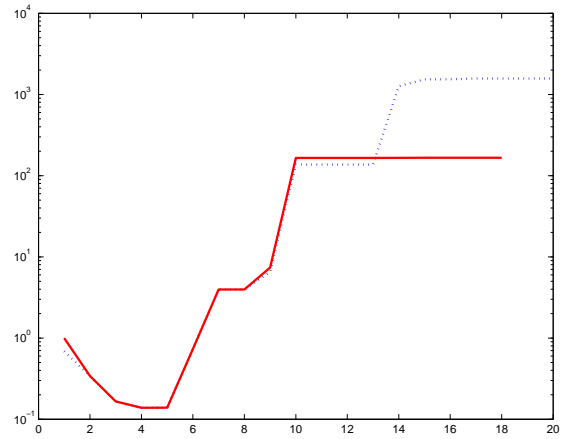

(b)

FIG. 4.6. Example 4.5: Noise-level $\nu=1 \times 10^{-2}$. (a) Differences $\left\|t_{k}-x_{k}\right\|$ for $k=2,3,4, \ldots$. (b) Relative errors $\left\|x_{k}-\hat{x}\right\| /\|\hat{x}\|$ for LSQR (blue dashed curve) and relative errors $\left\|t_{k}-\hat{x}\right\| /\|\hat{x}\|$ for RRE-LSQR (red solid curve).

responding extrapolated vectors $t_{k}$. This difference is minimal for $k=5$. We therefore choose the truncation index $k_{\text {extrapol }}=5$. Figure $4.6(\mathrm{~b})$ and Table 4.6 show $k_{\text {extrapol }}=5$ to be a suitable truncation index. In this example both $\left\|x_{k}-\hat{x}\right\|$ and $\left\|t_{k}-\hat{x}\right\|$ grow with $k$ for $k \geq 5$.

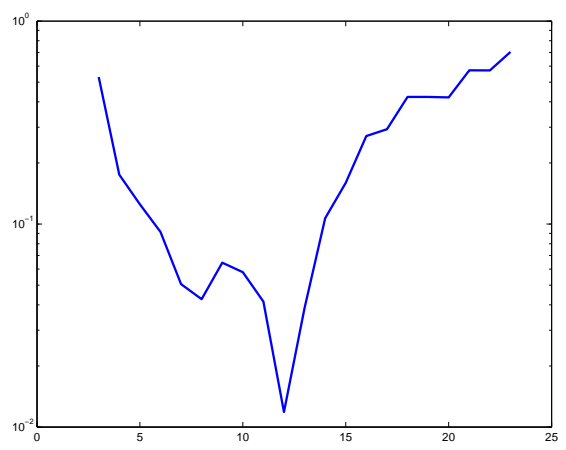

(a)

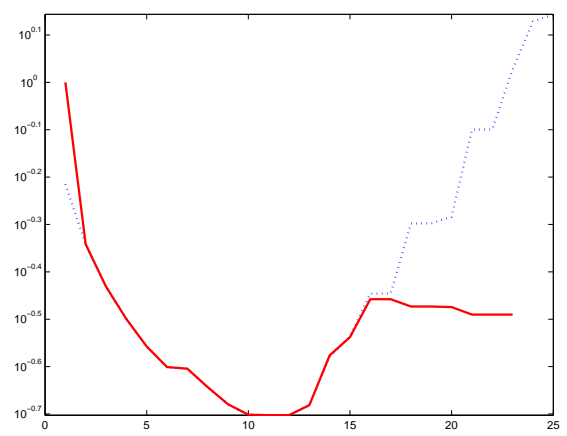

(b)

FIG. 4.7. Example 4.6: Noise-level $\nu=1 \times 10^{-2}$. (a) Differences $\left\|t_{k}-x_{k}\right\|$ for $k=2,3,4, \ldots$. (b) Relative errors $\left\|x_{k}-\hat{x}\right\| /\|\hat{x}\|$ for LSQR (blue dashed curve) and relative errors $\left\|t_{k}-\hat{x}\right\| /\|\hat{x}\|$ for $R R E-L S Q R$ (red solid curve).

Example 4.6. The matrix $A \in \mathbb{R}^{1000 \times 1000}$ and solution $\hat{x} \in \mathbb{R}^{1000}$ are generated with the code deriv2 from [20] with the linear solution. The underlying integral 


\begin{tabular}{ccc}
$k$ & $\left\|x_{k}-\hat{x}\right\| /\|\hat{x}\|$ & $\left\|t_{k}-\hat{x}\right\| /\|\hat{x}\|$ \\
\hline 4 & $1.39 \times 10^{-1}$ & $1.39 \times 10^{-1}$ \\
5 & $1.38 \times 10^{-1}$ & $1.38 \times 10^{-1}$ \\
6 & $7.33 \times 10^{-1}$ & $7.33 \times 10^{-1}$ \\
& \multicolumn{2}{c}{ TABLE 4.6}
\end{tabular}

Example 4.5: Noise-level $\nu=1 \times 10^{-2}$. Errors in LSQR solutions $x_{k}$ and in extrapolated LSQR solutions $t_{k}$.

\begin{tabular}{ccc}
$k$ & $\left\|x_{k}-\hat{x}\right\| /\|\hat{x}\|$ & $\left\|t_{k}-\hat{x}\right\| /\|\hat{x}\|$ \\
\hline 10 & $1.99 \times 10^{-1}$ & $1.99 \times 10^{-1}$ \\
11 & $1.99 \times 10^{-1}$ & $1.99 \times 10^{-1}$ \\
12 & $1.98 \times 10^{-1}$ & $1.98 \times 10^{-1}$ \\
13 & $2.08 \times 10^{-1}$ & $2.08 \times 10^{-1}$ \\
& \multicolumn{2}{c}{ TABLE 4.7}
\end{tabular}

Example 4.6: Noise-level $\nu=1 \times 10^{-2}$. Errors in LSQR solutions $x_{k}$ and in extrapolated $L S Q R$ solutions $t_{k}$.

equation is described in [25, p. 310]. Figure 4.7(a) shows the norm of the difference between the LSQR iterates $x_{k}$ and the corresponding extrapolated approximate solutions $t_{k}$. This difference is minimal for $k=12$. Figure 4.7(b) shows the iterate $x_{12}$ to be a fairly accurate approximation of $\hat{x}$. In fact, Table 4.7 shows $x_{12}$ to be the best approximation of $\hat{x}$.

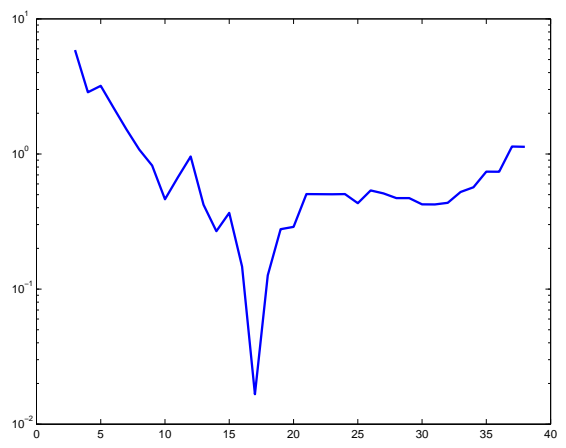

(a)

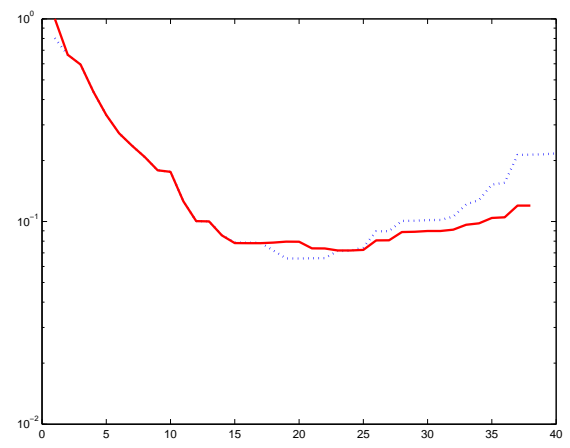

(b)

FIG. 4.8. Example 4.7: Noise-level $\nu=1 \times 10^{-2}$. (a) Differences $\left\|t_{k}-x_{k}\right\|$ for $k=2,3,4, \ldots$. (b) Relative errors $\left\|x_{k}-\hat{x}\right\| /\|\hat{x}\|$ for LSQR (blue dashed curve) and relative errors $\left\|t_{k}-\hat{x}\right\| /\|\hat{x}\|$ for $R R E-L S Q R$ (red solid curve).

Example 4.7. This example determines approximate solutions to the linear discrete ill-posed problems defined by the MATLAB code heat from [20]. The matrix $A \in \mathbb{R}^{1000 \times 1000}$ is nonsymmetric. The error in $b \in \mathbb{R}^{1000}$ corresponds to the noise-level $\nu=1 \times 10^{-2}$. The underlying ill-posed problem is described by Carasso [26].

Figure 4.8(a) displays the norm of the difference between the LSQR iterates $x_{k}$ and the corresponding extrapolated approximate solutions $t_{k}$. This difference is minimal for $k=17$. Thus, we choose the truncation index $k_{\text {extrapol }}=17$. Figure $4.8(\mathrm{~b})$ shows the iterate $x_{17}$ to be a fairly accurate approximation of $\hat{x}$. The best approximation is furnished by $x_{18}$; see Table 4.8 .

Example 4.8. The previous examples illustrate that extrapolation can determine 


\begin{tabular}{ccc}
$k$ & $\left\|x_{k}-\hat{x}\right\| /\|\hat{x}\|$ & $\left\|t_{k}-\hat{x}\right\| /\|\hat{x}\|$ \\
\hline 17 & $7.33 \times 10^{-2}$ & $7.35 \times 10^{-2}$ \\
18 & $7.21 \times 10^{-2}$ & $7.23 \times 10^{-2}$ \\
19 & $7.58 \times 10^{-2}$ & $7.78 \times 10^{-2}$ \\
20 & $7.61 \times 10^{-3}$ & $7.82 \times 10^{-2}$ \\
\multicolumn{3}{c}{ TABLE 4.8}
\end{tabular}

Example 4.7: Noise-level $\nu=1 \times 10^{-2}$. Errors in LSQR solutions $x_{k}$ and in extrapolated LSQR solutions $t_{k}$.

\begin{tabular}{lccc} 
Problem & $\nu=1 \times 10^{-2}$ & $\nu=1 \times 10^{-3}$ & $\nu=1 \times 10^{-4}$ \\
\hline shaw & $0 \%$ & $0 \%$ & $0 \%$ \\
baart & $0 \%$ & $0 \%$ & $0 \%$ \\
wing & $0 \%$ & $0 \%$ & $0 \%$ \\
foxgood & $2 \%$ & $0 \%$ & $0 \%$
\end{tabular}

TABLE 4.9

Example 4.8: Percentage of "failures" of the extrapolation method for computing the truncation index for TSVD for three noise-levels and several test problems from [20].

optimal or near-optimal truncation indices for many problems. This and the following examples shed light on the average performance of this approach to determine the truncation index when applied to several test problems from [20]. We measure the average performance similarly as in [7].

We consider the performance of TSVD. The truncation index $k_{\text {extrapol }}$ is determined by extrapolation as described in Example 4.1 and $k_{\text {opt }}$ is defined by (2.4). Column two of Table 4.9 displays how many times (in percent, rounded to one significant digit) the inequality

$$
\left\|x_{k_{\text {extrapol }}}-\hat{x}\right\|>10\left\|x_{k_{\mathrm{opt}}}-\hat{x}\right\|
$$

holds over 100 runs with matrices $A \in \mathbb{R}^{50 \times 50}$ and random noise with noise-level $\nu=1 \times 10^{-2}$. We refer to the situation when (4.2) holds as a "failure." Results for the noise-levels $\nu=1 \times 10^{-3}$ and $\nu=1 \times 10^{-4}$ are shown in columns three and four, respectively. The small entries in columns two through four indicate that the determination of the truncation index by extrapolation is quite reliable.

Example 4.9. Our last example provides results equivalent to column three of Table 4.9 when the approximate solution is determined with LSQR instead of with TSVD. The inequality (4.2) holds $0 \%$ (over 100 runs) for the problems shaw, deriv2 with a linear solution, and heat. For baart, the inequality (4.2) holds in $8 \%$ of the runs. We conclude that our method for choosing the truncation index for LSQR works well for many problems.

5. Conclusion. This paper describes a new criterion based on vector extrapolation for determining a suitable truncation index $k_{\text {extrapol }}$ for TSVD and LSQR. The extrapolation criterion has been applied to the solution of numerous discrete ill-posed problems for different noise-levels. Some of the computed results are reported in Section 4. The good performance of the extrapolation criterion suggests that $k_{\text {extrapol }}$ may be a valuable indicator of when to truncate, which can be applied either by itself or together with one or several other heuristic truncation criteria. Generally, $x_{k_{\text {extrapol }}}$ is a more accurate approximation of $\hat{x}$ than the corresponding extrapolated approximation $t_{k_{\text {extrapol }}}$. 


\section{REFERENCES}

[1] H. W. Engl, M. Hanke, and A. Neubauer, Regularization of Inverse Problems, Kluwer, Dordrecht, 1996.

[2] P. C. Hansen, Rank-Deficient and Discrete Ill-Posed Problems, SIAM, Philadelphia, 1998.

[3] A. Bouhamidi, K. Jbilou, L. Reichel, and H. Sadok, An extrapolated TSVD method for linear discrete ill-posed problems with Kronecker structure, Linear Algebra Appl., 434 (2011), pp. $1677-1688$.

[4] K. Jbilou, L. Reichel, and H. Sadok, Vector extrapolation enhanced TSVD for linear discrete ill-posed problems, Numer. Algorithms, 51 (2009), pp. 195-208.

[5] S. Kindermann, Convergence analysis of minimization-based noise level-free parameter choice rules for linear ill-posed problems, Electron. Trans. Numer. Anal., 38 (2011), pp. 233-257.

[6] F. Bauer and M. A. Lukas, Comparing parameter choice methods for regularization of ill-posed problem, Math. Comput. Simulation, 81 (2011), pp. 1795-1841.

[7] L. Reichel and G. Rodriguez, Old and new parameter choice rules for discrete ill-posed problems, Numer. Algorithms, 63 (2013), pp. 65-87.

[8] C. Brezinski, M. Redivo Zaglia, G. Rodriguez, and S. Seatzu, Extrapolation techniques for ill-conditioned linear systems, Numer. Math., 81 (1998), pp. 1-29.

[9] C. Brezinski, G. Rodriguez, and S. Seatzu, Error estimates for linear systems with applications to regularization, Numer. Algorithms, 49 (2008), pp. 85-104.

[10] C. Brezinski, G. Rodriguez, and S. Seatzu, Error estimates for the regularization of least squares problems, Numer. Algorithms, 51 (2009), pp. 61-76.

[11] L. Reichel, G. Rodriguez, and S. Seatzu, Error estimates for large-scale ill-posed problems, Numer. Algorithms, 51 (2009), pp. 341-361.

[12] C. Brezinski and M. Redivo Zaglia, Extrapolation Methods: Theory and Practice, NorthHolland, Amsterdam, 1991.

[13] S. Duminil and H. Sadok, Reduced rank extrapolation applied to electronic structure computations, Electron. Trans. Numer. Anal., 38 (2011), pp. 347-362.

[14] K. Jbilou and H. Sadok, Vector extrapolation methods. Applications and numerical comparison, J. Comput. Appl. Math., 122 (2000), pp. 149-165.

[15] C. C. Paige and M. A. Saunders, LSQR: An algorithm for sparse linear equations and sparse least squares, ACM Trans. Math. Software, 8 (1982), pp. 43-71.

[16] G. H. Golub and C. F. Van Loan, Matrix Computations, 3rd ed., Johns Hopkins University Press, Baltimore, 1996.

[17] R. P. Eddy, Extrapolation to the limit of a vector sequence, in Information Linkage Between Applied Mathematics and Industry, ed. P. C. C. Wang, Academic Press, New York, 1979 pp. 387-396.

[18] M. Mesina, Convergence acceleration for the iterative solution of $x=A x+f$, Comput. Meth. Appl. Mech. Eng., 10 (1977), pp. 165-173.

[19] W. D. Ford and A. Sidi, Recursive algorithms for vector extrapolation methods, Appl. Numer. Math., 4 (1988), pp. 477-489.

[20] P. C. Hansen, Regularization tools version 4.0 for MATLAB 7.3, Numer. Algorithms, 46 (2007), pp. 189-194.

[21] M. L. Baart, The use of auto-correlation for pseudo-rank determination in noisy ill-conditioned least-squares problems, IMA J. Numer. Anal., 2 (1982), pp. 241-247.

[22] G. M. Wing, A Primer on Integral Equations of the First Kind, SIAM, Philadelphia, 1991.

[23] C. T. H. Baker, The Numerical Treatment of Integral Equations, Clarendon Press, Oxford, 1977.

[24] C. B. Shaw, Jr., Improvements of the resolution of an instrument by numerical solution of an integral equation, J. Math. Anal. Appl. 37 (1972), pp. 83-112.

[25] L. M. Delves and J. L. Mohamed, Computational Methods for Integral Equations, Cambridge University Press, Cambridge, 1985.

[26] A. S. Carasso, Determining surface temperatures from interior observations, SIAM J. Appl. Math., 42 (1982), pp. 558-574. 